\title{
CARACTERÍSTICAS HISTOPATOLÓGICAS DE LA GLÁNDULA PROSTÁTICA EN CANINOS MAYORES DE CINCO AÑOS
}

\author{
David Ravelo O. ${ }^{1}$, Luis Tabacchi N. ${ }^{1}$, Rosa Perales C. ${ }^{1}$ y Gilberto Santillán A. ${ }^{1}$
}

\section{Whiturtert}

Prostate glands from 40 canines aged 5 years or greater, submitted to the Histopathology Laboratory of the San Marcos University Faculty of Veterinary Medicine, were studied. Macroscopic evaluation was made, the glands were measured (length and width in $\mathrm{cm}$ ) and weighed ( $\mathrm{g}$ ), and the age and body weight of the animals recorded. Histological sections of external (Corpus prostatae) and internal parts (Pars disseminata prostatae) related to the urethral lumen of the glands, were prepared using hematoxylin, eosin, Picro Ponceau, Manson trichrome and Van Gieson stains. Microscopic evaluation revealed hyperplastic, inflammatory, degenerative and neoplastic alterations. Hyperplastic processes were found in 33 of the 40 cases $(82.5 \%)$, with glandular hyperplasia present in 12 cases $(36 \%)$ and cystic glandular hyperplasia in 21 cases $(64 \%)$. Inflammatory processes (chronic non-suppurative prostatitis) were observed in $12.5 \%(5 / 40)$ of the glands. One degenerative process case $(2.5 \%)$ was diagnosed as glandular atrophy and one neoplastic process case $(2.5 \%)$ was diagnosed as adenocarcinoma. The prostate weight (gr) / body weight $(\mathrm{kg})$ ratio ranged between 0.62 and 8.32 in the hyperplastic cases, between 0.85 and 1.90 in the inflammatory alterations, was of 0.60 in the atrophy case and 1.65 in the neoplastic alteration. Twelve specimens showed bladder hypertrophy, corresponding to $30 \%(12 / 40)$ of the cases studied.

Key words: Prostate, glandular hyperplasia, cystic glandular hyperplasia, prostatitis, canine.

Se realizó el estudio histopatológico de la glándula prostática en 40 caninos mayores de 5 años de edad, remitidos para su necropsia al Laboratorio de Histopatología de la Facultad de Medicina Veterinaria de la UNMSM. Se hizo la evaluación macroscópica y se tomó medidas (largo $\mathrm{x}$ ancho en $\mathrm{cm}$ ), peso (g) de la glándula, edad del animal (años) y peso corporal $(\mathrm{kg})$; para establecer un Índice Prostático. La evaluación histológica se realizó tomándose muestras de la porción externa (corpus prostatae) y de la porción interna (Pars disseminata prostatae) relacionada a la luz uretral. Se utilizó las coloraciones de Hematoxilina Eosina, Picro Ponceau, Tricromico de Masson y Van Giesson. Se realizó el diagnostico histopatológico y se clasificó las siguientes alteraciones: hiperplásicas, inflamatorias, degenerativas y neoplásicas. Se diagnostico hiperplasia en un total de 33 casos que corresponden al $82.5 \%$ (33/40), de los cuales 12 , correspondientes al $36 \%$ (12/ $33)$, se identificaron como Hiperplasia glándular y 21 casos correspondientes al $64 \%$ (21/ 33) se diagnosticaron como hiperplasia glándular quística. Se identificó 5 casos correspondientes a proceso inflamatorios, los cuales fueron diagnosticados como Prostatitis

\footnotetext{
'Laboratorio de Histopatologia - FMV - UNMSM. Apdo. 41-0068 Lima-Perú.
} 
no supurativa crónica, que representó el $12.5 \%$ (5/40) del total de casos estudiados. Como procesos degenerativos, fue diagnosticado 1 caso como Atrofia glandular lo que representa el $2.5 \%$ (1/40). Dentro de las neoplasias, se diagnosticó 1 caso como Adenocarcinoma, que correspondió al 2.5\% (1/40) de los casos estudiados. El índice prostático en los casos de hiperplasia estuvo en los rangos de 0.62 y $8.32 \mathrm{~g}$. En el caso de atrofia fue de $0.60 \mathrm{~g}$. En la alteración neoplásica fue de $1.65 \mathrm{~g}$ y en las alteraciones inflamatorias fueron entre 0.85 y $1.90 \mathrm{~g}$. En 12 caninos se encontró hipertrofia de la vejiga que correspondió al $30 \%$ (12/40) del total de casos estudiados.

Palabras clave: Próstata, hiperplasia glandular, hiperplasia glándular quística, prostatitis.

\section{Intinimetrit}

Las glándulas sexuales accesorias en los machos, tales como la próstata, las glándulas vesiculares y las bulbouretrales, están íntimamente relacionadas con la uretra pélvica y difieren entre las distintas especies. La próstata esta presente en todos los mamíferos domésticos, algunas especies solo tienen el cuerpo (caballo), otras solo la parte diseminada (pequeños rumiantes) y las demás tienen ambas. La glándula prostática en el canino juega un papel esencial por ser la única glándula genital accesoria. Esta glándula en el perro se encuentra más desarrollada y presenta más alteraciones que en otras especies (Miller, 1991).

La función glandular prostática es importante debido a que provee un medio nutricional a los espermatozoides. Las enfermedades de la glándula prostática en el canino son comparables a las observadas en el hombre (Jones y Hunt, 1990). En el perro, al igual que en el hombre, las lesiones degenerativas (hiperplasia prostática) y neoplásicas son alteraciones que se presentan con mayor frecuencia en adultos mayores de 5 años (Dahme y Weiss, 1989).

En un estudio anatomopatológico de la glándula prostática en caninos realizado en nuestro medio y cuyas edades fluctuaban de 2 meses hasta 15 años de edad, se halló que el tamaño y peso de la próstata varia según la edad; y que los casos de enfermedades de la glándula prostática era manifiesta con mayor frecuencia en los animales mayores de 5 años (Tabacchi y Carpio 1994).
El presente trabajo tiene como objetivo determinar las características de las alteraciones histopatológicas de la glándula prostática en caninos mayores de cinco años, y establecer datos referenciales para la práctica clínica médico veterinaria.

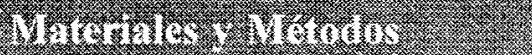

\section{Obtención de los especímenes:}

Las muestras se obtuvieron de 40 caninos machos enteros, mayores de 5 años, que fueron remitidos para su necropsia al Laboratorio de Histopatología de la Facultad de Medicina Veterinaria de la UNMSM. Se tomaron como caso todos los animales sin importar si estos presentaban historia clínica. Se evaluó la edad y peso del animal.

\section{Evaluación de la Glándula Prostática:}

Extraída la glándula prostática se procedió a evaluar el peso, aspecto macroscópico $\mathrm{y}$ las medidas de la glándula (largo $\mathrm{x}$ ancho), anotándose en una ficha los datos obtenidos. Se procedió a realizar una evaluación del peso de la próstata y del peso del animal para obtener un valor indicativo (peso de la glándula/peso corporal) que se expresó en g de tejido glándular por $\mathrm{kg}$ de peso, para establecer un índice prostático del crecimiento de la glándula y su relación con cuadros de hiperplasia benigna los cuales fueron corroborados microscópicamente.

Para la evaluación microscópica: se tomaron muestras de dos áreas de la próstata: de la porción externa (Corpus prostatae) 
y de la porción interna (Pars disseminata prostatae), relacionada a la luz de la uretra prostática, las cuales fueron recogidas y depositadas en un frasco de vidrio conteniendo una solución fijadora de formol al $10 \%$ para su procesamiento histopatológico.

\section{Clasificación y diagnóstico de las altera- ciones:}

Una vez obtenidas las descripciones y diagnósticos histopatológicos, se procedió a clasificar las alteraciones como sigue:

- Hiperplásicas.

- Inflamatorias.

- Degenerativas.

- Neoplásicas.

\section{$153017+106$}

Los resultados se detallan en el Cuadro 1.

\section{Procesos Hiperplásicos}

Se diagnosticaron un total de 33 casos, que corresponden al $82.5 \%$ (33/40), de los cuales 12 casos fueron identificados como Hiperplasia glandular prostática (HG) que representan el $36 \%$ (12/33), caracterizada por una proliferación del epitelio glandular y proyecciones papilares en la porción externa (Corpus prostatae), existiendo una proliferación glandular menor y escasa proliferación fibromuscular en la porción interna (Pars disseminata prostatae) con relación a la uretra prostática cuyo epitelio no mostraba áreas de descamación ni reducción significativa de la luz.

Se diagnosticaron 21 casos correspondientes a Hiperplasia glándular quística (HGQ); que representó el 64\% (21/33); caracterizada por la proliferación en la porción externa (Corpus prostatae) del epitelio glandular y dilatación de los acinos glandulares con proyecciones papilares con presencia de múltiples cavidades quísticas, en las que se observó una sustancia hialina que corresponde al fluído prostático, así como la presencia de linfocitos y moderada fibrosis. En la porción interna (Pars disseminata prostatae), la hiperplasia glandular predominaba sobre las formaciones quísticas, existiendo una mayor infiltración linfocitaria y fibrosis, sin afectar el epitelio uretral ni la luz del conducto.

Macroscópicamente, el órgano esta aumentado de tamaño, la simetría de los lóbulos es variable, al corte se observan formaciones quísticas de tamaño conocidas con el nombre de "panal de abeja".

La Figura 1 muestra la frecuencia de casos de Hiperplasia glandular prostática (HG) e Hiperplasia glandular quística (HGQ).

\section{Procesos Inflamatorios}

Se identificaron 5 casos correspondientes a prostatitis no supurativa crónica, que representan el $12.5 \%(5 / 40)$; caracterizado en la porción externa (Corpus prostatae), por descamación del epitelio glandular, infiltración de linfocitos, células plasmáticas y macrófagos, se observa áreas importantes de fibrosis y neoformación vascular. En la porción interna (Pars disseminata prostatae), así como infiltración linfocitaria por debajo del epitelio uretral y descamación del mismo, en esta porción la fibrosis es importante, se observa también focos hemorrágicos en pequeñas zonas de la porción interna.

\section{Procesos Degenerativos}

Se identificó 1 caso compatible con cuadro de atrofia glandular prostática, que corresponde al $2.5 \%$ (1/40); caracterizado por la infiltración de tejido fibromuscular alrededor de los espacios intersticiales del epitelio glandular, con reducción del tamaño de los acinos y su reemplazo progresivo por áreas de fibrosis, esto se evidencia claramente en la porción interna (Pars disseminata prostatae). En la porción externa (Corpus prostatae), se observaron acinos glandulares 
Cuadro 1. Tabla de evaluación macroscópica y resultados anatomopatológicos $(n=40)$.

\begin{tabular}{|c|c|c|c|c|c|c|c|}
\hline \multirow{2}{*}{$\begin{array}{l}\text { Edad } \\
\text { (años) }\end{array}$} & \multirow{2}{*}{$\begin{array}{l}\text { Peso } \\
(\mathrm{kg})\end{array}$} & \multirow[t]{2}{*}{ Raza } & \multicolumn{2}{|c|}{ Próstata } & \multirow{2}{*}{$\begin{array}{l}\text { Indice } \\
\text { Prostatico }\end{array}$} & \multirow{2}{*}{$\begin{array}{c}\text { Diagnostico } \\
\text { Histopatologico }\end{array}$} & \multirow{2}{*}{$\begin{array}{l}\text { Hipertrofia } \\
\text { Vesical }\end{array}$} \\
\hline & & & $\begin{array}{c}\text { Largo } \mathrm{x} \\
\text { ancho, } \mathrm{cm}\end{array}$ & Peso, $\mathrm{g}$ & & & \\
\hline 6 & 13.8 & cruzado & $2.0 \times 2.7$ & 9.0 & 0.65 & HG & $\left(^{*}\right)$ \\
\hline 6 & 78 & Fila & $6.4 \times 5.2$ & 82.4 & 1.06 & $\mathrm{HG}$ & $(*)$ \\
\hline 6 & 38 & Boxer & $4.0 \times 4.5$ & 52.0 & 1.36 & $\mathrm{HGQ}$ & $(*)$ \\
\hline 6 & 46 & Pastor alemán & $6.6 \times 5.4$ & 102.5 & 2.22 & $\mathrm{HGQ}$ & $(* *)$ \\
\hline 6 & 14 & Schnauzer & $4.0 \times 4.0$ & 40 & 2.85 & HGQ & $(*)$ \\
\hline 6 & 23 & Pastor alemán & $5.0 \times 4.2$ & 47.8 & 2.07 & $\mathrm{HG}$ & $(*)$ \\
\hline 7 & 24 & cruzado & $4.5 \times 5.0$ & 76.5 & 3.18 & HGQ & $(*)$ \\
\hline 7 & 7.5 & cruzado & $1.6 \times 3.0$ & 11.8 & 1.57 & $\mathrm{HGQ}$ & $\left(^{*}\right)$ \\
\hline 7 & 10 & Rottweiller & $6.0 \times 5.2$ & 83.2 & 8.32 & $\mathrm{HGQ}$ & $(*)$ \\
\hline 7 & 7.5 & Pequines & $1.7 \times 1.5$ & 6.0 & 0.85 & $\mathrm{PnSC}$ & $(*)$ \\
\hline 8 & 20 & cruzado & $2.7 \times 2.5$ & 15.5 & 0.77 & $\mathrm{HG}$ & $\left(^{*}\right)$ \\
\hline 8 & 18 & cruzado & $2.5 \times 3.0$ & 13.0 & 0.72 & $\mathrm{HG}$ & $(*)$ \\
\hline 8 & 55 & cruzado & $5.6 \times 5.0$ & 90.9 & 1.65 & $\begin{array}{l}\text { Adenocarcinoma } \\
\text { Prostatico }\end{array}$ & $(* *)$ \\
\hline 8 & 7.5 & cruzado & $3.5 \times 4.0$ & 42.4 & 5.65 & HGQ & $(* *)$ \\
\hline 8 & 10 & cruzado & $2.7 \times 3.5$ & 16.0 & 1.10 & $\mathrm{HG}$ & $(*)$ \\
\hline 8 & 7 & cruzado & $2.5 \times 3.0$ & 13.0 & 1.85 & HGQ & $(*)$ \\
\hline 8 & 24 & cruzado & $3.6 \times 3.0$ & 32.4 & 1.35 & $\mathrm{HG}$ & $(*)$ \\
\hline 9 & 18 & cruzado & $4.7 \times 5.7$ & 67.0 & 3.72 & $\mathrm{HGQ}$ & $(*)$ \\
\hline 9 & 13 & cruzado & $4.1 \times 3.4$ & 21.9 & 1.68 & $\mathrm{HG}$ & $(*)$ \\
\hline 9 & 12 & cruzado & $3.0 \times 2.5$ & 11.2 & 0.93 & $\mathrm{HG}$ & $(* *)$ \\
\hline 9 & 8 & cruzado & $1.7 \times 2.8$ & 14.0 & 1.75 & $\mathrm{HGQ}$ & $(*)$ \\
\hline 10 & 45 & cruzado & $4.8 \times 5.0$ & 37.0 & 0.82 & $\mathrm{HG}$ & $\left(^{*}\right)$ \\
\hline 10 & 26.5 & Samoyedo & $7.0 \times 5.5$ & 115.0 & 4.33 & $\mathrm{HGQ}$ & $(* *)$ \\
\hline 10 & 7.0 & cruzado & $4.4 \times 3.4$ & 32.5 & 4.64 & $\mathrm{HGQ}$ & $(*)$ \\
\hline 10 & 13 & Cocker & $3.2 \times 3.8$ & 26.0 & 2.0 & $\mathrm{HGQ}$ & $\left(^{*}\right)$ \\
\hline 10 & 13 & Cocker & $3.5 \times 2.5$ & 11.2 & 0.86 & $\mathrm{HG}$ & $(* *)$ \\
\hline 10 & 15 & cruzado & $4.5 \times 3.5$ & 23.5 & 1.56 & $\mathrm{PnSC}$ & $(*)$ \\
\hline 10 & 20 & cruzado & $2.5 \times 3.0$ & 12.1 & 0.60 & $\begin{array}{c}\text { Atrofia } \\
\text { Prostatica }\end{array}$ & $(* *)$ \\
\hline 11 & 16 & cruzado & $3.6 \times 4.5$ & 28.0 & 1.75 & HGQ & $(*)$ \\
\hline 11 & 40 & Rottweiller & $5.4 \times 4.3$ & 81.2 & 2.03 & $\mathrm{HG}$ & $(* *)$ \\
\hline 12 & 20 & Doberman & $4.5 \times 5.5$ & 48.8 & 2.44 & $\mathrm{HGQ}$ & $(*)$ \\
\hline 12 & 30 & Doberman & $3.8 \times 5.0$ & 57.0 & 1.90 & PnSC & $(*)$ \\
\hline 12 & 25 & cruzado & $3.0 \times 4.0$ & 27.5 & 1.10 & $\mathrm{HG}$ & $\left({ }^{*}\right)$ \\
\hline 13 & 8 & cruzado & $3.5 \times 3.2$ & 22.0 & 2.75 & $\mathrm{HGQ}$ & $(* *)$ \\
\hline 13 & 12 & cruzado & $6.4 \times 6.5$ & 95.5 & 7.95 & $\mathrm{HGQ}$ & $(* *)$ \\
\hline 15 & 8 & cruzado & $4.0 \times 5.2$ & 49.1 & 6.13 & $\mathrm{HGQ}$ & $(* *)$ \\
\hline 15 & 16 & cruzado & $3.6 \times 4.6$ & 85.0 & 5.31 & $\mathrm{HGQ}$ & $\left(^{*}\right)$ \\
\hline 15 & 8 & cruzado & $3.5 \times 3.5$ & 35.5 & 4.44 & $\mathrm{HGQ}$ & $\left(^{*}\right)$ \\
\hline 15 & 35 & cruzado & $3.8 \times 4.0$ & 32.8 & 0.93 & $\mathrm{HG}$ & $\left(^{*}\right)$ \\
\hline 17 & 15 & cruzado & $3.5 \times 3.6$ & 27.6 & 1.84 & $\mathrm{HGQ}$ & $(* *)$ \\
\hline
\end{tabular}

HG: Hiperplasia Glandular; HGQ: Hiperplasia Glandular Quistica; PnSC: Prostatitis no Supurativa Crónica; *: No presenta Hipetrofia Vesical; ${ }^{* *}$ : Si presenta Hipertrofia Vesical. 


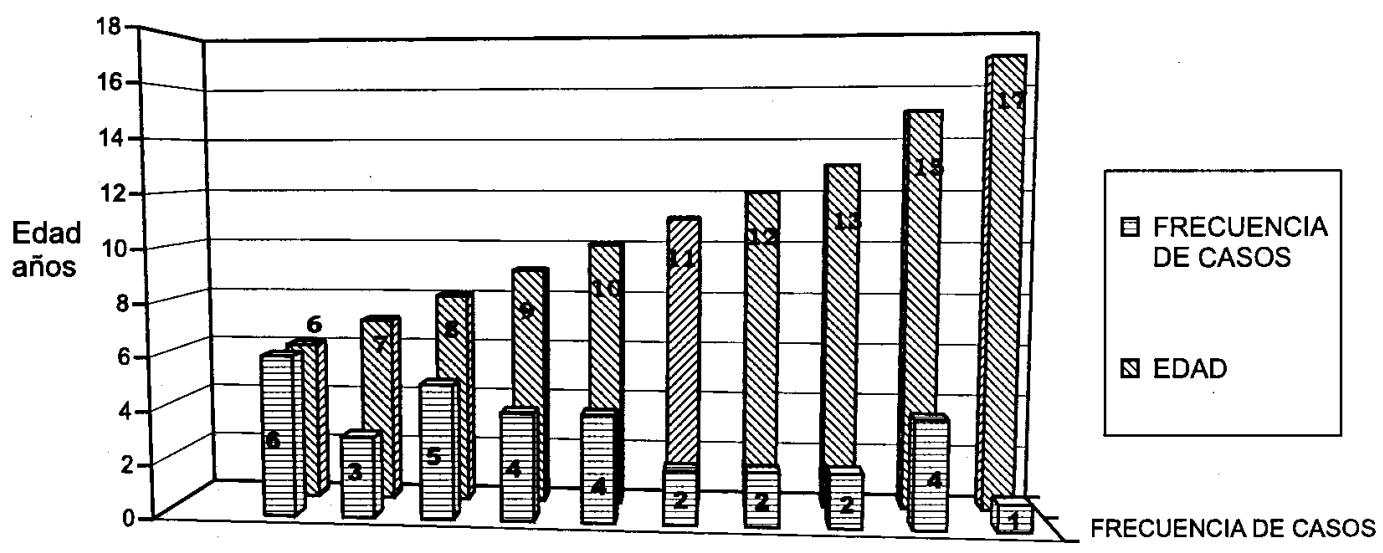

Figura 1. Frecuencia de casos de hiperplasia prostática por edades.

pequeños y epitelio glandular normal. Macroscópicamente la glándula es pequeña, de consistencia compacta y dura al corte.

\section{Procesos neoplásicos.}

Se diagnosticó 1 caso correspondiente a un adenocarcinoma prostático de tipo alveolar, que correspondió al 2.5\% (1/40); caracterizado por formaciones de células redondas de nucleo voluminoso y escaso citoplasma con varios nucleolos, dispuestos en estructuras semejantes a los alvéolos de la glándula prostática, así mismo se observó proliferación de tejido fibroso en la periferia del proceso neoplásico.

\section{Índice prostático y hallazgos patológicos:}

Se observó que los diagnósticos de los procesos correspondientes a HG y HGQ, estaban relacionadas con los casos en que el Índice Prostático, estaba comprendido entre los rangos de 0.62 hasta $8.32 \mathrm{~g}$.

En los casos correspondientes a Prostatitis no supurativa crónica el Índice Prostático estuvo entre los rangos de 0.85 y $1.90 \mathrm{~g} / \mathrm{kg}$.

El Índice Prostático en el caso diagnosticado como Atrofia prostática fue de $\mathbf{0 . 6 0}$ $\mathrm{kg}$ y en el caso de Adenocarcinoma prostático fue de 1.65 .
Se observó que 12 caninos presentaron a la necropsia procesos de hipertrofia de la capa muscular de la vejiga, lo que corresponde al $30 \%(12 / 40)$ de los casos estudiados.

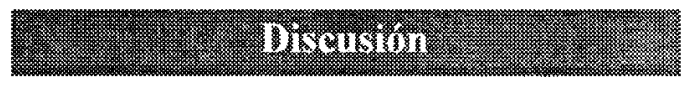

En el presente estudio, de las 40 muestras observadas histopatológicamente, la alteración más saltante son HG y HGQ que alcanzaron el 82.5\% (33/40) del total de casos, porcentaje muy cercano al $85 \%$ mencionado por Dahme y Weiss (1989), en caninos mayores de 5 años, pero muy superior al $60 \%$ estimado por Johnston y Feeney (1991), en caninos de la misma edad. Se puede afirmar que la hiperplasia prostática es un proceso asociado al envejecimiento, que en mayor o menor grado esta presente en casi todo animal al llegar a cierta edad (mayores de 5 años).

Del total de muestras estudiadas, a la necropsia no se observó problemas de obstrucción de la uretra prostática, sin embargo en 12 casos se observaron hipertrofia de la capa muscular de la vejiga (Cuadro 1). El estudio microscópico confirmó que la hipertrofia vesical no fue causada directamente por el crecimiento de la glándula que pudiera obstruir la luz uretral. Además, el examen histopatológico reveló que el mayor grado de hiperplasia se encontró en la porción externa 
(Corpus prostatae), sin mayor compromiso de la porción interna (Pars disseminatta distalis); en ésta se observo una moderada hiperplasia glandular y proliferación fibromuscular sin afectar la integridad del epitelio de la uretra prostática ni la viabilidad de la misma. Podemos afirmar entonces, que la hiperplasia prostática no actúa directamente en la causa de estenosis de la uretra prostática y la consecuente hipertrofia vesical. Esto puede explicarse según Jubb y Kennedy (1991), que en el perro la uretra no esta completamente rodeada por la próstata, y que la presión de la glándula hiperplásica en la porción sacra del parasimpático a nivel de la pelvis produce paresia de la vejiga. De otro lado, la disminución de la luz uretral por estiramiento longitudinal contribuye al problema cuando la próstata hiperplásica se desplaza hacia delante y arriba de la cavidad pélvica. Peña y Domínguez (1997), mencionan que este fenómeno produce un desplazamiento de la vejiga cranealmente, provocando cuadros de distensión y atonía vesical e infecciones recurrentes del tracto urinario en casos avanzados.

En el hombre, la hipertrofia vesical como efecto de la hiperplasia prostática tiene su origen en la estructura anatómica de la próstata que rodea completamente la uretra y que en los casos de hiperplasia, esta ocurre a nivel de las glándulas submucosas y mucosas del lóbulo medio y obstruyen la luz uretral provocando dificultades graves en la emisión de orina, el resultado es las producción de una vejiga hipertrófica (Andersen, 1981). En contraste, este fenómeno se observa rara vez en el perro debido a que esta especie tiene mucho menor cantidad de tejido prostático rodeando la uretra y el crecimiento hiperplásico de este tiende a ocurrir alejándose de la misma (porción externa), además el lóbulo medio a comparación del hombre, falta o esta muy poco desarrollado en el perro (Peña y Domínguez, 1997).

Dahme y Weiss (1989), menciona que las dificultades para la emisión de heces a consecuencia de la hiperplasia prostática son debido a que por acción de la prensa abdominal, la próstata penetra en la cavidad pelviana comprimiendo el recto. Peña y Domínguez (1997), mencionan al "Prostatismo o síndrome Prostático" a la hiperplasia prostática, la cual es acompañada de síntomas digestivos en primer término, locomotores y urinarios en casos avanzados.

Podemos afirmar que si bien el canino es usado como modelo para el estudio de distintas patologías prostáticas humanas como el cáncer prostático (Andrawiss y Opolon, 1998), en el caso de la hiperplasia glandular el origen de ésta y la manera como se desarrolla son distintos en el hombre y el canino, por lo que se debe hacer la distinción respectiva para una mejor comprensión de los cuadros patológicos que se desprenden de estos procesos.

Existen divergencias en el uso del término "Hipertrofia" e "Hiperplasia" cuando se refiere a la glándula prostática. Dahme y Weiss (1989) usa el término de hipertrofia de la próstata y nos da una definición que caracteriza los fenómenos hiperplásicos sin hacer la aclaración respectiva; Jubb y Kennedy (1991) hace la misma mención y usa el término "Hipertrofia" para luego hablar de "Hiperplasia prostática" y de sus características. Peña y Domínguez (1997), utilizan el término de "Hiperplasia Prostática Benigna" o de "Hiperplasia Glanduloquística" y remarcan que la hiperplasia glandular se manifiesta en forma de hiperplasia epitelial sin hipertrofia. En el examen histológico de las muestras diagnosticadas como procesos hiperplásicos revelan que la definición de Hiperplasia prostática es la correcta y debe ser usada, en lugar del término de "hipertrofia de la próstata" que es una definición incorrecta.

La evaluación de los 33 casos diagnosticados como alteraciones hiperplásicas y la relación entre el peso de la próstata y el peso corporal de los mismos, mostró un valor de $0.65 \mathrm{~g}$ como valor mínimo y como máximo de $8.32 \mathrm{~g}$. Este valor usado como Índice Prostático es próximo al hallado por Schlotthauer y Bollman (1936), 
que sugirieron $0.70 \mathrm{~kg}$ como valor máximo es considerado normal para una glándula prostática en perros menores de 5 años. Ellos sugieren que la relación peso de la próstata y peso corporal es constante hasta los 5 años y a partir de esa edad la glándula comienza a incrementar gradualmente su peso. Esto ofrece un valor indicativo favorable del crecimiento de la glándula y su relación con cuadros de hiperplasias corroborados microscópicamente.

Tabacchi y Carpio (1994), estudiaron las características anatomopatológicas de la próstata de 24 caninos cuyas edades variaban entre 2 meses hasta 15 años y utilizaron el índice prostático sugerido por Schlotthauer y Bollman (1936) encontrando una relación significativa entre el peso de la glándula prostática y el peso corporal de los perros siendo más manifiesto en los animales mayores de 5 años, los mismos que en su mayoría se ve afectados por procesos patológicos.

Con relación a los fenómenos inflamatorios, Tabacchi y Carpio (1994), en caninos de edades de 6 a 15 años encontraron 6 casos correspondientes a cuadros de prostatitis crónica ( 3 supurativos y 3 no supurativos), cuyas edades estaban comprendidas entre 8 y 14 años. En nuestro estudio se observó 5 casos de prostatitis no supurativa crónica en caninos cuyas edades estuvieron comprendidas entre 7 y 12 años. La similitud de los rangos de edad encontrados en ambos casos nos sugiere un proceso característico de perros geriátricos que no son detectados a tiempo y que tienden a la cronicidad. Esta afección es habitualmente supurativa y produce abscesos. Una prostatitis crónica es dificil de tratary a veces en los animales es asintomático y no causa problemas para la eyaculación.

Sólo se observó un caso de Atrofia prostática en un canino de 10 años, cuyo índice prostático fue de $0.60 \mathrm{~kg}$ y presentaba una hipertrofia vesical, estas alteraciones degenerativas son características de animales geriátricos sin actividad sexual permanente o que no son machos dominantes, donde el declive de la actividad hormonal principalmente de la testosterona, induce una disminución de la actividad de la glándula y la instalación de una fibrosis progresiva. Schlotthauer y Bollman (1942) mencionan que la atrofia también ocurre en los animales castrados sin importar la edad. Es importante recordar que el desarrollo y mantenimiento de la actividad de la próstata depende de la presencia de testículos.

El hallazgo de una caso de Adenocarcinoma de tipo alveolar en un perro de 8 años con un índice prostático de 1.65 y una hipertrofia vesical importante, pone en evidencia una neoplasia primaria de la próstata en perros por encima de diez años (Jubb y Kennedy, 1991). Si bien es cierto se encontró esta neoplasia en una glándula donde había zonas de hiperplásica; Jubb y Kennedy (1991), señalan que la hiperplasia prostática no se transforma en neoplasias en los perros.

\section{connermot}

- En el presente estudio histopatológico, la alteración más importante es la Hiperplasia Glandular (HG) y la Hiperplasia Glandular Quística (HGQ) que alcanzó el 82.5\% (33/40) de los casos.

- La utilización de un Índice Prostático para evaluar el aumento de la glándula y su relación con cuadros de Hiperplasia prostática fue significativa, esto se corroboró microscópicamente.

- La Hipertrofia de la Vejiga no tiene como origen directo las alteraciones Hiperplásica, por el contrario los trastornos digestivos en relación con la obstrucción rectal son causa directa de estas por su estrecha relación anatómica.

- Se recomienda una evaluación clínica preventiva de la próstata en caninos a partir de 5 años para poder establecer diagnósticos precoces, los cuales pueden ser apoyados por exámenes ecográficos, radiológicos y citológicos. 


\section{Etcrain Cinth}

1. Andersen, W. 1981. Anatomia Patológica Basica. $9^{\text {a }}$ Ed, P. 377-379. Editorial Doyma S.A. España.

2. Andrawiss, M. y P. Opolon. 1998. Transfert de gène par adénovirus dans la prostate de chien: Implications pour la thérapie génique du cancer de la prostate. Communications Département d'urologie, Hôpital Saint-Louis, 75475 Paris, France.

3. Dahme, E. y E. Weiss. 1989. Anatomía Patología Especial Veterinaria. 3ra. Ed. p. 257-260.Editorial Acribia. España.

4. Johnston, G y D. Feeney. 1991. Diagnostics of Male Canine Reproductive Organs - Methods and Limitations- Veterinary Clinics of North America: Small Animal Practice, 21(3), p. 553-589. U.S.A.

5. Jones, C. y R. Hunt. 1990. Patologia Veterinaria. Vol 3. $1^{a}$ Ed. p. 1598-1602 Editoral Hemisferio Sur. Argentina.
6. Jubb, F. y C. Kennedy. 1991. Patología de los Animales Domésticos. Vol 3. 3ra. Ed. p. 552-558. Editorial Hemisferio Sur. Argentina.

7. Miller, M. 1991. Disección del Perro. $3^{\mathrm{a}}$ Ed. p. 180-181 Editorial Interamericana Mc Graw Hill. España.

8. Peña, F. y J. Dominguez. 1997. Hiperplasia Prostática en el perro. Archivos de Reproducción Animal, Publicación Anual. p. 72 - 77.

9. Schlotthauer, C. y J. Bollman. 1936. The Prostate Gland of Dog. The Cornell Veterinarian. Vol.\# 4. p. 343-350. U.S.A.

10. Schlotthauer, C. y J. Bollman. 1942. The Effect of Artificial Cryptorchidism on the Prostate Gland of Dog. Am. J. Vet. Res. 8: p.151. U.S.A.

11. Tabacchi, L. y J. Carpio. 1994. Estudio Anatomopatológico de la glándula prostática en el perro. Rev. Teorema revista de Investigación de la UNMSM. Año $3 . \mathrm{N}^{\circ}$ 5. Octubre 94. p. 35 - 36. Perú. 